\title{
Constraints on WIMP annihilation for contracted Dark Matter in the inner Galaxy with gamma-rays
}

\author{
Aldo Morselli* \\ INFN Roma Tor Vergata, Italy \\ E-mail: aldo.morsellieroma2.infn.it
}

\begin{abstract}
Detection of gamma rays and cosmic rays from the annihilation or decay of dark matter particles is a promising method for identifying dark matter, understanding its intrinsic properties, and mapping its distribution in the universe. Based on $N$-body simulations the largest $\gamma$-ray signal from DM annihilation is expected from the centre of the Galaxy. In the same region a large $\gamma$-ray background is produced by bright discrete sources and the cosmic-rays interacting with the interstellar gas and the photons fields but the DM-induced gamma-ray emission is expected to be so large there that the search is still worthwhile. We derive constraints on parameters of generic dark matter candidates by comparing theoretical predictions with the gamma-ray emission observed by the Fermi-LAT from the region around the Galactic Center. Our analysis is conservative since it simply requires that the expected dark matter signal does not exceed the observed emission.
\end{abstract}

Science with the New Generation of High Energy Gamma-ray experiments, 10th Workshop 04-06 June 2014

Lisbon - Portugal

* Speaker. 
Astrophysical searches for dark matter (DM) are a fundamental part of the experimental efforts to explore the dark sector. The strategy is to search for DM annihilation products in preferred regions of the sky, i.e., those with the highest expected DM concentrations and still close enough to yield high DM-induced fluxes at the Earth. For that reason, the Galactic Center (GC), nearby dwarf spheroidal galaxy (dSphs) satellites of the Milky Way, as well as local galaxy clusters are thought to be among the most promising objects for DM searches. In particular, dSphs represent very attractive targets because they are highly DM-dominated systems and are expected to be free from any other astrophysical gamma-ray emitters that might contaminate any potential DM signal. Although the expected signal cannot be as large as that from the GC, dSphs may produce a larger signal-to-noise $(S / N)$ ratio. This fact allows us to place very competitive upper limits on the gamma-ray signal from DM annihilation [1, 2, 3], using data collected by the Large Area Telescope (LAT) onboard the Fermi gamma-ray observatory [4]. These are often referred to as the most stringent limits on DM annihilation cross-section obtained so far.

Despite these interesting limits derived from dSphs, the GC is still expected to be the brightest source of DM annihilations in the gamma-ray sky by several orders of magnitude. Although several astrophysical processes at work in the crowded GC region make it extremely difficult to disentangle the DM signal from conventional emissions, the DM-induced gamma-ray emission is expected to be so large there that the search is still worthwhile. Furthermore, the DM density in the GC may be larger than what is typically obtained in $N$-body cosmological simulations. Ordinary matter (baryons) dominates the central region of our Galaxy [5]. Thus, baryons may significantly affect the DM distribution. As baryons collapse and move to the center they increase the gravitational potential, which in turn forces the DM to contract and increase its density. This is a known and qualitatively well understood physical process $[6,8,9]$. It is also observed in many cosmological simulations that include hydrodynamics and star formation [10,11, 12, 13, 14, 15]. If this is the only effect of baryons, then the expected annihilation signal will substantially increase $[5,7]$.

A preliminary analysis of Fermi LAT observations of the GC region was presented in [16], [17]. In [18] we analyze in detail the constraints that can be obtained for generic DM candidates from Fermi-LAT inner Galaxy gamma-ray measurements assuming some specific (and well motivated) DM distributions. The approach is conservative, requiring simply that the expected DM signal does not exceed the gamma-ray emission observed by the Fermi-LAT in an optimized region around the GC. The region is chosen in such a way that the $S / N$ ratio is maximized. This kind of analysis, without modeling of the astrophysical background, was also carried out by the Fermi-LAT collaboration to constrain DM models from Galactic halo observations [19].

The results are presented in Figure 1, where the constraints obtained are shown for different final states. There we also illustrate the case $\langle\sigma v\rangle=3 \times 10^{-26} \mathrm{~cm}^{3} \mathrm{~s}^{-1}$, which corresponds to the value of the annihilation cross-section associated to the correct thermal relic abundance for a WIMP whose annihilation is dominated by the s-wave (velocity-independent) contribution and thus, $\Omega_{D M} h^{2} \approx 3 \times 10^{-27} \mathrm{~cm}^{3} \mathrm{~s}^{-1}\langle\sigma v\rangle^{-1} \approx 0.1$ [21]. For comparison, the constraints are given considering only the contribution from prompt gamma rays and the total contribution from prompt plus ICS gamma rays.

First, it is worth noting that if the DM density follows an Einasto, NFW or Burkert profile, the upper limits on the annihilation cross section are above the value of the thermal cross-section for any annihilation channel. Nevertheless, the situation is drastically different when we consider 

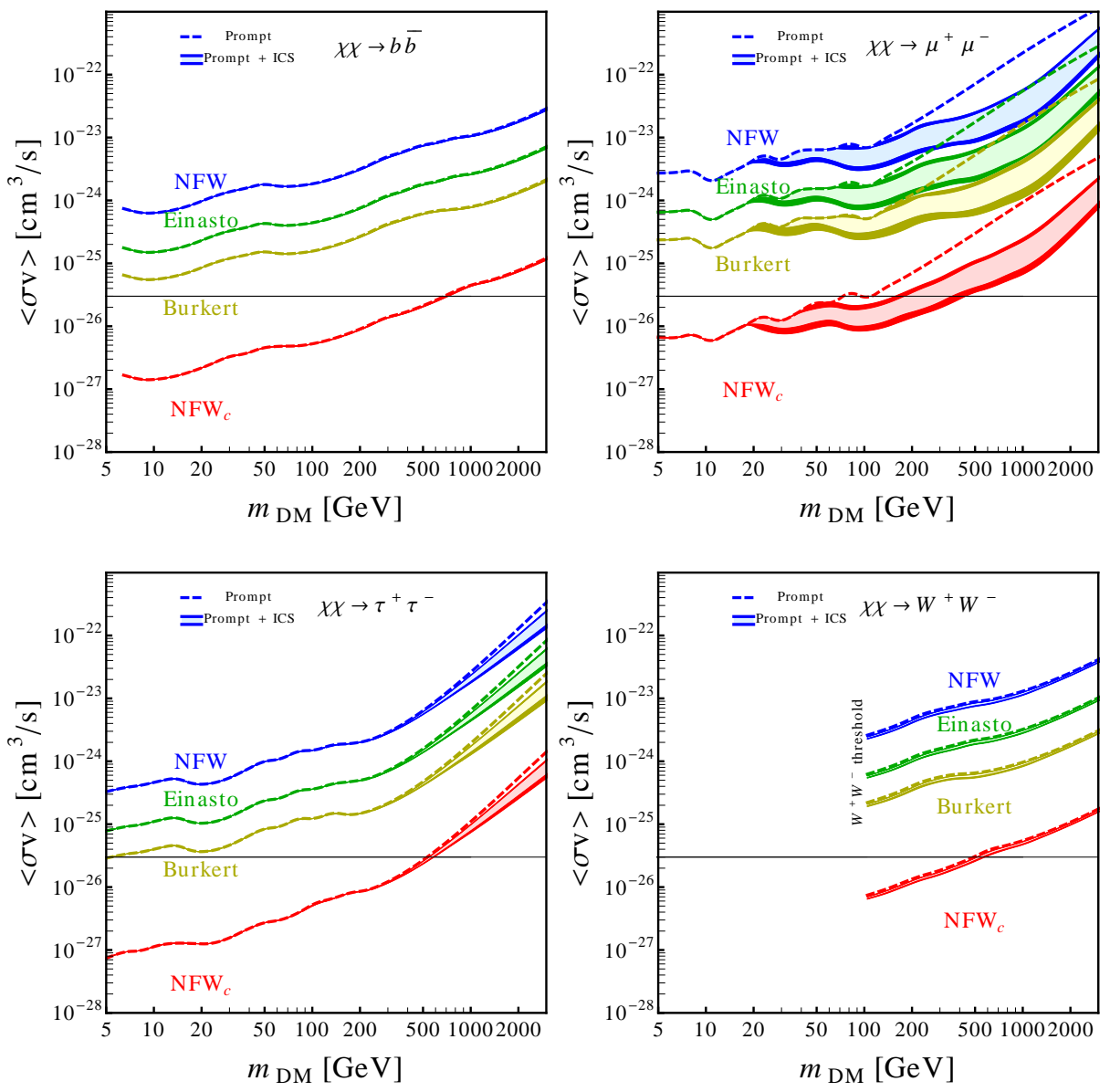

Figure 1: $3 \sigma$ upper limits on the annihilation cross-section of models in which DM annihilates into $b \bar{b}$, $\mu^{+} \mu^{-}$(upper panel), $\tau^{+} \tau^{-}$or $W^{+} W^{-}$(lower panel), for the four DM density profiles discussed in the text. Upper limits set without including the ICS component in the computation are also given as dashed curves (prompt) for comparison. The uncertainty in the diffusion model is shown as the thickness of the solid curves (from top to bottom: MIN, MED, MAX) while the lighter shaded regions represent the impact of the different strengths of the Galactic magnetic field with lower(higher) values of the cross-section corresponding to $B_{0}=1 \mu \mathrm{G}\left(B_{0}=10 \mu \mathrm{G}\right)$. The horizontal line corresponds to the expected value of the thermal cross-section for a generic WIMP candidate.

the DM compression due to baryonic infall in the inner region of the Galaxy. As pointed out in Ref. [5], the effect of the baryonic adiabatic compression might be crucial for indirect DM searches, as it increases by several orders of magnitude the gamma-ray flux from DM annihilation in the inner regions, and therefore the DM detectability. Indeed, by adopting the $\mathrm{NFW}_{c}$ profile and for a $b \bar{b}, \tau^{+} \tau^{-}$and $W^{+} W^{-}$channel, the thermal annihilation cross-section is already reached for a DM mass of 680,530 and $490 \mathrm{GeV}$, respectively. For the $\mu^{+} \mu^{-}$channel the effect of the prompt gamma rays is less important since generally fewer photons are produced in the FSR compared to the hadronic decays of the other channels. (For the $W^{+} W^{-}$which is open when $m_{D M} \gtrsim 90 \mathrm{GeV}$, the $W^{ \pm}$decays produce a large number of photons, especially at high energy). Notice that the lower bound associated with prompt gamma rays for $\mu^{+} \mu^{-}$is $100 \mathrm{GeV}$ compared to about 500$700 \mathrm{GeV}$ in the other channels. Thus the ICS is important in this case, also due to the relatively 


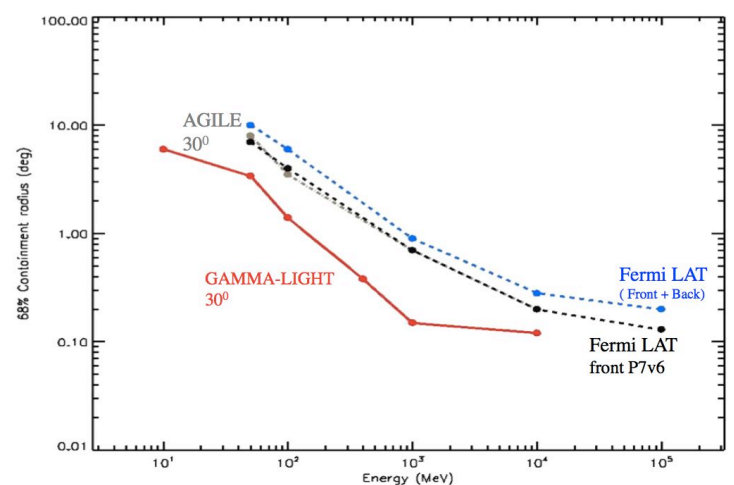

Figure 2: Point Spread Function (PSF, 68\% containment radius) of the GAMMA-LIGHT gamma-ray (GRID) imager (in red color) obtained by extensive GEANT-4 simulations which assume an incidence angle of $30^{\circ}$, Silicon strip analog readout, and Kalman filter analysis of particle tracks. For comparison, we show the Fermi-LAT Pass7V6 PSF (total LAT: blue curve; front-LAT: black color) and the AGILE PSF (in gray color).

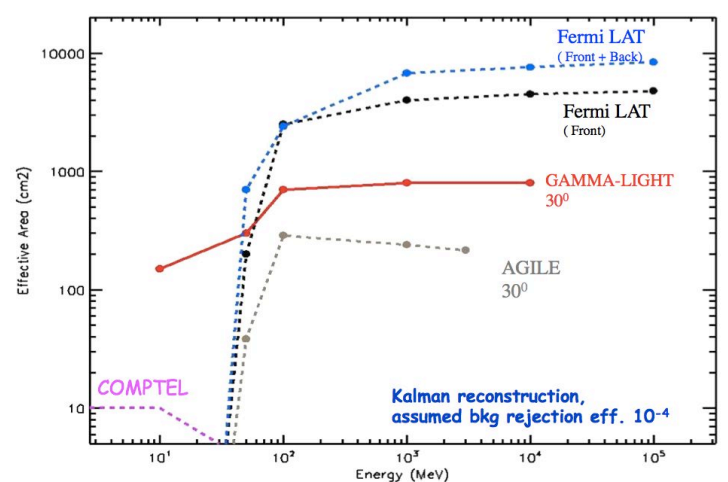

Figure 3: Effective area for the GAMMA-LIGHT GRID at 30 degree off-axis (in red color). For comparison, we also show the effective areas of AGILE at 30 degree off-axis (in gray color), Fermi-LAT-front Pass7 V6 at normal incidence (total: blue color; front-LAT: black color), and COMPTEL's (in purple). Trigger logic efficiency and background rejection have been taken into account.

harder $e^{ \pm}$spectrum [20]. We can see that for $B_{0}=1 \mu \mathrm{G}$ the lower bound on the DM mass turns out to be $358 \mathrm{GeV}$ and for $B_{0}=10 \mu \mathrm{G}$ the bound is $157 \mathrm{GeV}$, using the MIN diffusion model. For MED and MAX diffusion models the values turn out to be 404, $171 \mathrm{GeV}$ and $439,179 \mathrm{GeV}$, respectively. As discussed in [18], when the magnetic field is stronger the energy of the injected $e^{ \pm}$ is more efficiently liberated in the form of microwaves, resulting in a softer gamma-ray spectrum, and producing therefore lower constraints. Therefore, we have shown that in those cases in which the ICS component is dominant (for heavy WIMP masses in general), the variation of the magnetic field can significantly alter the expected gamma-ray fluxes from the inner regions of the Galaxy.

Although the above results can be interpreted in general as implying that vanilla WIMP models and contracted DM profiles are incompatible with the Fermi data, one should keep in mind that if one works in the framework of a specific particle physics model this conclusion might in principle be avoided in some regions of the parameter space. For example, the final state can be 


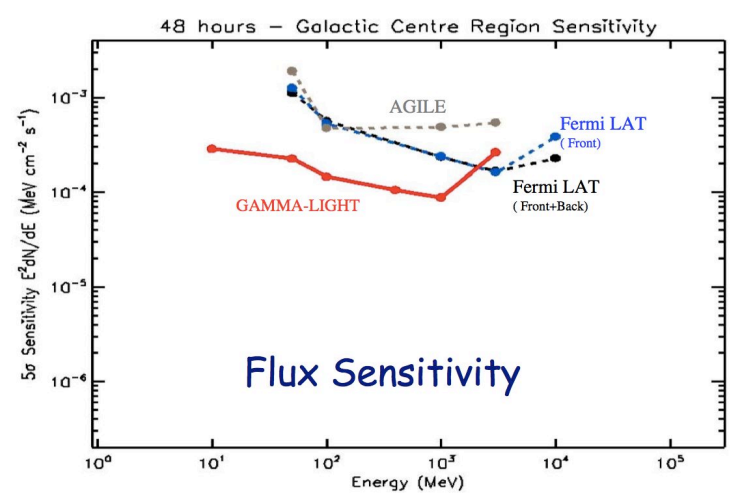

Figure 4: Point source (5-sigma) sensitivity for $48 \mathrm{hr}$ (solar time) observation at $30^{\circ}$ off-axis of the GAMMA-LIGHT GRID imager (in red color). Also shown are the Fermi-LAT Pass7V6 sensitivity (totalLAT: black color; front-LAT: blue color) and AGILE's sensitivity (gray) for the same duration.

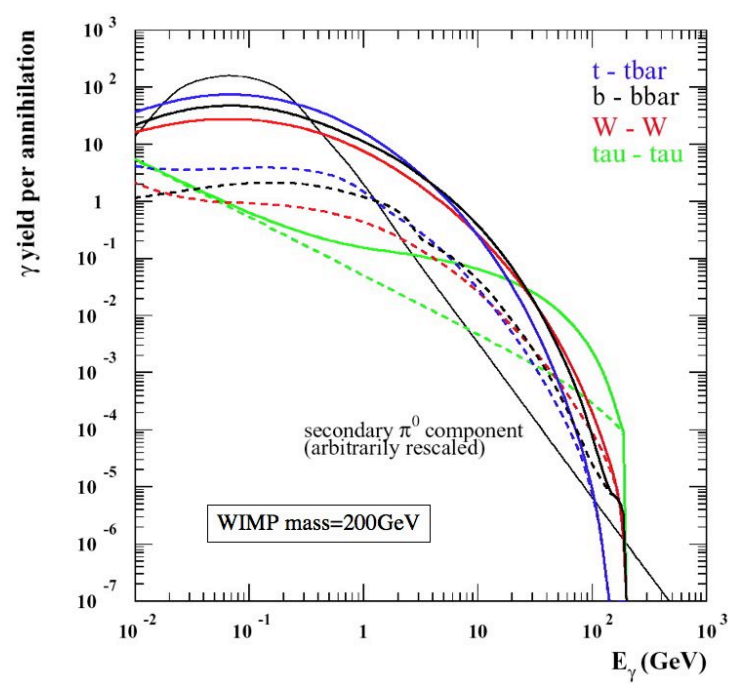

Figure 5: Differential energy spectra per annihilation for a few sample annihilation channels and a fixed WIMP mass $(200 \mathrm{GeV})$.

a combination of the annihilation channels presented here, as in supersymmetry where the lightest neutralino annihilation modes are $70 \% \bar{b} b-30 \% \bar{\tau} \tau$ for a Bino DM, and $100 \% W^{+} W^{-}$for a Wino DM (or for a Higgs-portal model). More importantly, the value of the annihilation cross section in the Galactic halo might be smaller than $3 \times 10^{-26} \mathrm{~cm}^{3} \mathrm{~s}^{-1}$ for a DM candidate that is thermally produced. For example, in the early Universe coannihilation channels can also contribute to $\langle\sigma v\rangle$. Also, DM particles whose annihilation in the early Universe is dominated by p-wave (velocitydependent) contributions would have a smaller value of $\langle\sigma v\rangle$ in the Galactic halo, where the DM velocity is much smaller than at the time of freeze-out, and can therefore escape the constraints derived in this work. These two effects can in fact occur in some regions of the parameter space of well motivated models for particle DM, such as the neutralino. In this sense, the results derived above for pure annihilation channels can be interpreted as limiting cases that give an idea of what can happen in realistic scenarios. 


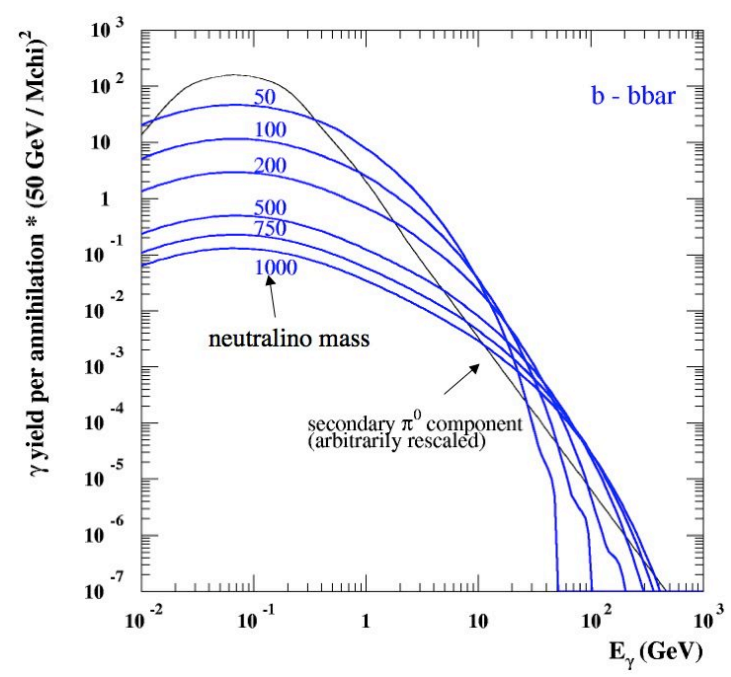

Figure 6: Differential $\gamma$-ray energy spectra per annihilation for a fixed annihilation channel (b bar) and for a few sample values of WIMP masses [23]. For comparison we also show the emissivity, with an arbitrarily rescaled normalization, from the interaction of primaries with the interstellar medium. The solid lines are the total yields, while the dashed lines are components not due to $\pi^{0}$ decays.

A new version of the event-level reconstruction and analysis framework (called Pass 8 ) is foreseen soon from the Fermi LAT collaboration. With this new analysis software we should increase the efficiency of the instrument at high energy and have a data set based on independent event analysis thus gaining a better control of the systematic effects.

At low energy (below $50 \mathrm{MeV}$ ) a new instrument like Gamma-Light [24] can really improve these results.

The Point Spread Function of Gamma-Light is shown in figure 2 , the effective area is shown in figure 3 and the sensitivity for $48 \mathrm{hr}$ (solar time) observation is shown in figure 4

The importance of Gamma-Light for Dark Matter searches can be seen in figures 6 and 5 where the differential $\gamma$-ray energy spectra per annihilation of Weakly Interacting Massive Particle (WIMP) are plotted [23]. As one can see the bulk of the emission even for high WIMP masses is in the energy range $5 \mathrm{MeV}-100 \mathrm{MeV}$.

Let us finally remark that decaying DM can produce a detectable line in the Gamma-Light energy range [22]. In principle, detectability is expected to be large in the very Galactic Center since hadronic emission models for this region are predicting a fall down about $100 \mathrm{MeV}$ (see Fig. 2 of [25]).

\section{References}

[1] M. Ackermann et al. [Fermi-LAT Collaboration], Phys. Rev. Lett. 107 (2011) 241302 [arXiv:1108.3546 [astro-ph.HE]].

[2] A. A. Abdo et al. [Fermi-LAT Collaboration], Astrophys. J. 712 (2010) 147 [arXiv:1001.4531 [astro-ph.CO]].

[3] A. Geringer-Sameth and S.M. Koushiappas, Phys. Rev. Lett. 107 (2011) 241303 [arXiv:1108.2914 [astro-ph.CO]]. 
[4] W. B. Atwood et al. [Fermi-LAT Collaboration], Astrophys. J. 697 (2009) 1071 [arXiv:0902.1089 [astro-ph.IM]].

[5] F. Prada, A. Klypin, J. Flix Molina, M. Martinez and E. Simonneau, Phys. Rev. Lett. 93 (2004) 241301 [astro-ph/0401512].

[6] Ya. B. Zeldovich, A. A. Klypin, M. Yu. Khlopov and V. M. Chechetkin, Sov. J. Nucl. Phys. 31 (1980) 664.

[7] Y. Mambrini, C. Muñoz, E. Nezri and F. Prada, JCAP 01 (2006) 010 [hep-ph/0506204].

[8] G.R. Blumenthal, S.M. Faber, R. Flores and J.R. Primack, Astrophys. J. 301 (1986) 27.

[9] O. Y. Gnedin, A. V. Kravtsov, A. A. Klypin and D. Nagai, Astrophys. J. 616 (2004) 16 [astro-ph/0406247 [astro-ph]].

[10] M. Gustafsson, M. Fairbairn and J. Sommer-Larsen, Phys. Rev. D 74 (2006) 123522 [astro-ph/0608634 [astro-ph]].

[11] P. Colín, O. Valenzuela and A. A. Klypin, Astrophys. J. 644 (2006) 687 [astro-ph/0506627 [astro-ph]].

[12] P. B. Tissera, S. D. M. White, S. Pedrosa and C. Scannapieco, MNRAS 406 (2010) 922 [arXiv:0911.2316 [astro-ph.CO]].

[13] O. Y. Gnedin, D. Ceverino, N. Y. Gnedin, A. A. Klypin, A. V. Kravtsov, R. Levine, D. Nagai and G. Yepes, arXiv:1108.5736 [astro-ph.CO].

[14] M. Zemp, O. Y. Gnedin, N. Y. Gnedin and A. V. Kravtsov, Astrophys. J. 748 (2012) 54 [arXiv:1108.5384 [astro-ph.GA]].

[15] J. Sommer-Larsen and M. Limousin, MNRAS 408 (2010) 1998 [arXiv:0906.0573 [astro-ph.CO]].

[16] V. Vitale and A. Morselli for the Fermi/LAT Collaboration, 2009 Fermi Symposium, eConf Proceedings C091122 [arXiv:0912.3828]

[17] A. Morselli, B.Cañadas, V.Vitale, Il Nuovo Cimento 34 C, N. 3 (2011) [arXiv:1012.2292]

[18] G.A.Gomez-Vargas et al. JCAP 10 (2013) 029 [arXiv:1308.3515]

[19] M. Ackermann et al. [Fermi LAT Collaboration], Astrophys. J. 761 (2012) 91 [arXiv:1205.6474 [astro-ph.CO]].

[20] A. Birkedal, K. T. Matchev, M. Perelstein and A. Spray, hep-ph/0507194.

[21] G. Jungman, M. Kamionkowski and K. Griest, Phys. Rep. 267 (1996) 195 [arXiv:hep-ph/9506380].

[22] G.A. Gómez-Vargas, M. Fornasa, F. Zandanel, A.J. Cuesta, C. Muñoz, F. Prada and G. Yepes, JCAP 02 (2012) 001 [arXiv:1110.3305].

[23] A.Cesarini, F.Fucito, A.Lionetto, A.Morselli, P. Ullio, Astropart. Phys. 21 (2004) 267 [astro-ph/0305075]

[24] A.Morselli et al., Nuclear Physics B - $239 Đ 240$ (2013) 193-198 [arXiv:1406.1071]

[25] T. Linden, E. Lovegrove and S. Profumo, arXiv:1203.3539 